Archive for

Organic Chemistry

Arkivoc 2018, part v, 0-0

\title{
Metal-free addition of aliphatic carboxylic acids to cyanopropargyl alcohols: an access to new families of functionalized dihydrofurans and $3(2 \mathrm{H})$-furanones
}

\author{
Olesya A. Shemyakina, ${ }^{a}$ Ol'ga G. Volostnykh, ${ }^{a}$ Anton V. Stepanov, ${ }^{a}$ Igor' A. Ushakov, ${ }^{a}$ Anastasiya G. \\ Mal'kina, ${ }^{a}$ Konstantin A. Apartsin, ${ }^{b, c}$ Viktoria V. Kireeva, ${ }^{c}$ and Boris A. Trofimov*a
}
${ }^{a}$ A. E. Favorsky Irkutsk Institute of Chemistry, Siberian Branch, Russian Academy of Sciences, 1 Favorsky Str., 664033, Irkutsk, Russian Federation. Fax: (395-2)41-93-46
${ }^{b}$ The Irkutsk Scientific Center of Surgery and Traumatology, 664003 Irkutsk, Russian Federation
${ }^{c}$ Biomedical Research and Technology Department of the Irkutsk Scientific Center, Siberian Branch, Russian Academy of Sciences, 664003 Irkutsk, Russian Federation
Email: boris trofimov@irioch.irk.ru

Received 03-14-2018

Accepted 04-26-2018

Published on line 06-06-2018

\section{Abstract}

The metal-free $\mathrm{Et}_{3} \mathrm{~N}$-mediated addition of cyanopropargyl alcohols to aliphatic carboxylic acids provides for straightforward efficient access to 4-cyano-[(Z)-3-cyanomethylene]-2,3-dihydrofurans and 4-cyano-3(2H)furanones of pharmaceutical value. By comparison to analogous reactions using aromatic and heteroaromatic carboxylic acids, this synthesis is implemented under much milder conditions (room temperature vs. $100{ }^{\circ} \mathrm{C}$ and microwave assistance) to give essentially higher relative content of 2,3-dihydrofurans (2:1 assembly products).
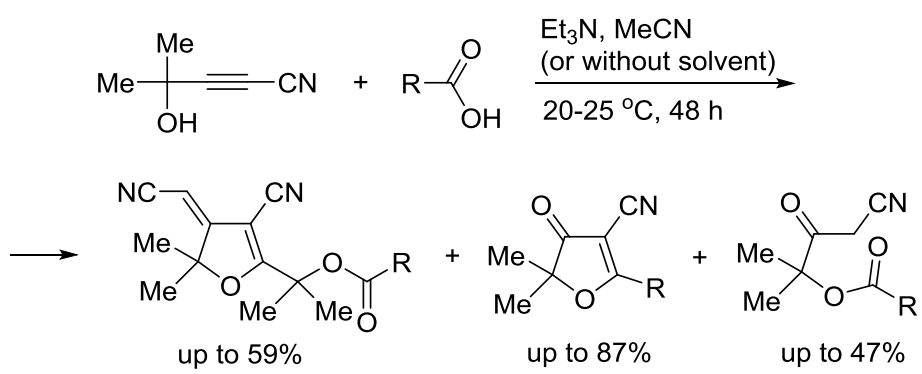

$\mathrm{R}=\mathrm{Me}, \mathrm{Pr}, \mathrm{Bu}, i-\mathrm{Pr}, i-\mathrm{Bu}$

Keywords: Tertiary cyanopropargyl alcohols, carboxylic acids, tandem reaction, cyclization, 2,3-dihydrofurans, $3(2 H)$-furanones 


\section{Introduction}

Functionalized derivatives of dihydrofurans are currently in focus of synthetic organic chemistry. ${ }^{1-3}$ These structures are closely related to the ribose fragment of nucleosides, consequently, DNA and other ribosetailored life-sustaining structures including diverse dehydro and deoxy sugars. ${ }^{4-6}$ Dihydrofuran scaffold is frequently met in natural products and pharmaceuticals. ${ }^{7-9}$ As a typical illustration, 2,3-dihydrofuran ring is a fragment of diterpenoids (bicunningine $A$ and $B$ ), isolated from a tree of traditional Chinese medicine used for the treatment of hernia, arthritis and strangury. ${ }^{10}$ Dihydrofuran derivatives are widely employed as versatile building blocks in synthetic organic chemistry. ${ }^{11}$ 2,3-Dihydrofurans are recognized as precursors for the asymmetric synthesis of tetrahydrofurans. ${ }^{12-16}$ Synthetic approaches to 2,3-dihydrofurans involve the [4+1] cycloaddition of enones with diazo compounds, ${ }^{17,18}$ the $[3+2]$ cyclization of aldehydes with $\beta$-ketosulfides/ $\beta$ ketosulfones. ${ }^{11,19-21}$ For this purpose, the ring closing reaction of 1,3-dicarbonyl compounds with alkenes is efficient as well. ${ }^{22-25}$ Cyclization of the but-3-yn-1-ols is also a method of choice for the synthesis of 2,3dihydrofurans. ${ }^{26-29}$ Recently, a method for the preparation of functionalized 2,3-dihydrofurans by $\mathrm{Sc}(\mathrm{OTf})_{3^{-}}$ catalyzed cyclization of $\alpha$-allylated 1,3-dicarbonyl compounds has been reported. ${ }^{11}$ The palladium-catalyzed reaction of aryl and heteroaryl bromides, chlorides, and nonaflates with $\alpha$-allyl- $\beta$-ketoesters provides a ready access to functionalized 2,3-dihydrofurans. ${ }^{30}$

Until now only a few examples of the reaction of carboxylic acids with acetylenes are known to finish at the addition step to give the functionalized 1,3-dienes and vinyl esters. ${ }^{31-35}$ These processes are usually catalyzed by transition-metal salts or complexes, e.g. silver ${ }^{31}$ and palladium ${ }^{32}$ acetate, (arene)(phosphine)ruthenium, ${ }^{33,34}$ polynuclear transition-metal-sulfur complexes \{palladium cuboidal cluster $\left[\mathrm{PdMo}_{3} \mathrm{~S}_{4}(\operatorname{tacn})_{3} \mathrm{Cl}\right]\left[\mathrm{PF}_{6}\right]_{3}$. $^{35} \mathrm{To}^{3}$ our knowledge, no basic catalysts have been successfully employed for the addition of carboxylic acids to acetylenes (except for the our works ${ }^{36-42}$ ). Recently, naphthylcarboxylic acids ${ }^{36}$ and a number of heterocyclic (thiophene, ${ }^{37}$ furan, ${ }^{38}$ pyrrole ${ }^{39-40}$ ) carboxylic acids have been shown to be also efficient in this synthesis, for the heterocyclic carboxylic acids a microwave assistance and a higher temperature $\left(100{ }^{\circ} \mathrm{C}\right)$ being required. The synthesis proves to be appropriate for some functionalized aromatic carboxylic acids (3-amino- ${ }^{41}$ and 3 hydroxybenzoic ${ }^{42}$ acids).

\section{Results and Discussion}

The aim of this work is to essentially extend the substrate scope of the above assembly and to increase structural diversity of the functionalized dihydrofurans thus found by involving aliphatic acids, yet another vast class of organic acids, into the reaction. Also, differences of this reaction as compared to the previous specific examples are intended to be examined. To reach this aim, available tertiary cyanopropargyl alcohol ${ }^{43}$ has $^{4}$ been allowed to react with $2 \mathrm{a}-\mathrm{e}$ in the presence of $\mathrm{Et}_{3} \mathrm{~N}$ (Scheme 1, Tables 1, 2).

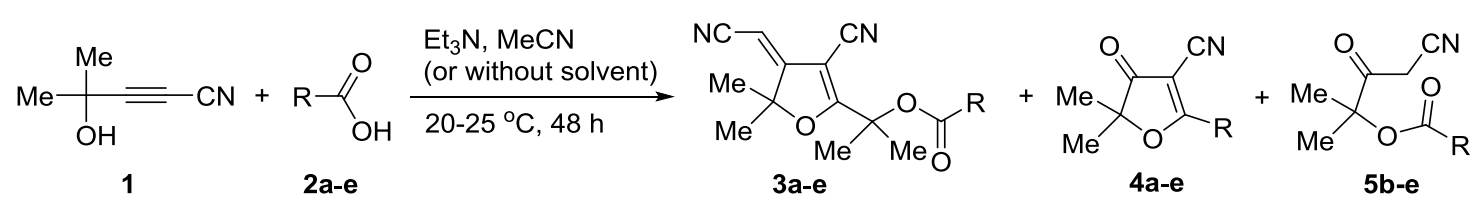

Scheme 1. A competing cyclization of tertiary cyanopropargyl alcohol $\mathbf{1}$ with aliphatic carboxylic acids $\mathbf{2 a - e .}$ 
The reaction was carried out both in solvent $(\mathrm{MeCN})$ and without solvent at a various reactants ratio. The better results were attained at room temperature and 1 equivalent of $\mathrm{Et}_{3} \mathrm{~N}$ relative to carboxylic acids. At a cyanopropargyl alcohol 1/aliphatic carboxylic acids 2 ratio of 1.2:1 in MeCN, the yields of 3(2H)-furanones 4a-e ranged $21-87 \%$. The yields of 2,3-dihydrofurans $3 a-e$ were $8-18 \%$ and those of intermediates $5 \mathbf{b}$-e spanned 14 47\% (Table 1).

Table 1. Products of the reaction between cyanopropargyl alcohol $\mathbf{1}$ and aliphatic carboxylic acids 2 a-e at reactant ratio of $1.2: 1$ correspondingly $\left(\mathrm{Et}_{3} \mathrm{~N}, \mathrm{MeCN}, 20-25^{\circ} \mathrm{C}, 48 \mathrm{~h}\right)$

Entry Acid 2

${ }^{\mathrm{a}}$ The molar ratio of $\mathbf{1 / 2}$ was $1: 1 ;{ }^{\mathrm{b}} \mathrm{By}{ }^{1} \mathrm{H} \mathrm{NMR}$; ${ }^{\mathrm{c}} 2 \mathrm{eq}$. of $\mathrm{Et}_{3} \mathrm{~N}$ was used; ${ }^{\mathrm{d}}$ The reaction was carried out at $\sim 80{ }^{\circ} \mathrm{C}$ for $9.5 \mathrm{~h}$.

When the reaction of pentanoic acid $\mathbf{2 c}$ and cyanopropargyl alcohol $\mathbf{1}$ was conducted with the excess $\mathrm{Et}_{3} \mathrm{~N}$ $(\mathrm{MeCN}, 48 \mathrm{~h}$ ), ratio and yields of products $\mathbf{3 c}, \mathbf{4 c}$ and $\mathbf{5 c}$ were insignificantly varied (Table 1 , entry 4 ). A higher temperature improved the chemoselectivity of product 4 . At heating $\left(\sim 80^{\circ} \mathrm{C}\right)$ the reaction of 3 -methylbutanoic acid $\mathbf{2 e}$ and cyanopropargyl alcohol 1 proceeded faster $(4 \mathrm{~h})$ but the content of product $\mathbf{5 e}$ in the reaction mixture was $\sim 40 \%\left({ }^{1} \mathrm{H} \mathrm{NMR}\right.$ data). Further heating $\left(\sim 80{ }^{\circ} \mathrm{C}\right)$ for $5.5 \mathrm{~h}$ afforded $3(2 \mathrm{H})$-furanone $4 \mathrm{e}$ in $57 \%$ preparative yield (Table 1, entry 7). Yield of keto ester 5 e decreased to 4\%. 2,3-Dihydrofuran 3 e was not isolated.

As seen from Table 2, when the two-fold molar excess of cyanopropargyl alcohol 1 relative to carboxylic acids 2 a-e was used, the product ratio changed in favor of 2,3-dihydrofurans 3a-e (27-59\%), 3(2H)-furanones 
4a-e and intermediates $\mathbf{5 b}$,c,e being minor products (6-20\% and 6-19\%, correspondingly). Unlike the reaction intended to obtain excessive amounts of $3(2 H)$-furanones $4 a-e$ (Table 1 ), these syntheses were implemented without solvent.

Table 2. Products of the reaction of cyanopropargyl alcohol $\mathbf{1}$ with aliphatic carboxylic acids 2 a-e at the reactant ratio of $2: 1$, respectively $\left(\mathrm{Et}_{3} \mathrm{~N}\right.$, without solvent, $\left.20-25^{\circ} \mathrm{C}, 48 \mathrm{~h}\right)$

Entry Acid 2

${ }^{\mathrm{a}} \mathrm{By}{ }^{1} \mathrm{H}$ NMR.

In fact, at greater excess of cyanopropargyl alcohol, the chemoselectivity of the assembly relative to 2,3dihydrofurans increases. For example, with three-fold molar excess of $\mathbf{1}$ to acetic acid $\mathbf{2 a}$, the yield of the corresponding 2,3-dihydrofuran $3 a$ reaches $42 \%$ and that of $3(2 H)$-furanone 4 a decreases to $9 \%$ (cf. Table 2 , entry 1). A remarkable feature of 2:1 assembly leading to 2,3-dihydrofurans 3a-e is the predominant Zconfiguration of their cyanoethenyl moiety, i.e. this process is stereoselective.

The differences of the assembly studied as compared to analogous syntheses with heteroaromatic carboxylic acids ${ }^{40}$ are much milder conditions (room temperature vs. $100{ }^{\circ} \mathrm{C}$ and microwave irradiation) and essentially higher contents of 2,3-dihydrofurans in the reaction products.

In all the cases, the reaction course was monitored using the IR spectroscopy by the disappearance of the bands at $2297 \mathrm{~cm}^{-1}(C=\mathrm{C}-\mathrm{C}=\mathrm{N})$ and appearance of the bands at $2229-2211 \mathrm{~cm}^{-1}(=\mathrm{C}-\mathrm{C} \equiv \mathrm{N})$ assigned to the starting and target compounds, correspondingly. All the products were easily separated and purified by column chromatography on silica gel.

The configurational assignment and the substituent location for the compounds 3a-e are based on 2D (NOESY, ${ }^{1} \mathrm{H}-{ }^{13} \mathrm{C} \mathrm{HSQC},{ }^{1} \mathrm{H}-{ }^{13} \mathrm{C} \mathrm{HMBC}$ ) NMR spectroscopy data. The NMR $\left({ }^{1} \mathrm{H},{ }^{13} \mathrm{C}\right)$ and IR data of 2,3- 
dihydrofurans 3 a-e as well as $3(2 H)$-furanones $4 a-e$ are consistent with their structure. In the ${ }^{1} \mathrm{H}$ NMR spectra of 3a-e, signals of the olefin protons are observed (5.09-5.10 ppm). In the ${ }^{13} \mathrm{C} \mathrm{NMR}$ spectra of $3 a-e$, characteristic signals of the carbons of the carbonyl (171.7-175.7) and cyano (111.6-116.2 ppm) groups are present. The IR spectra of 2,3-dihydrofurans 3a-e show two bands at 2223-2217 and $2212-2211 \mathrm{~cm}^{-1}$ assigned to two cyano groups, the band of the conjugated carbonyl function $\mathrm{C}=0$ appears at $1741-1713 \mathrm{~cm}^{-1}$. The absorption bands of the double bond $\mathrm{N}-\mathrm{C}=\mathrm{C}$ and the $\mathrm{C}=\mathrm{C}-\mathrm{CN}$ groups are observed at $1645-1623$ and 1593-1563 $\mathrm{cm}^{-1}$, correspondingly. In the ${ }^{1} \mathrm{H}$ NMR spectra of 4a-e, signals of the alkyl protons are present. In the ${ }^{13} \mathrm{C}$ NMR spectra of compounds 4a-e the carbonyl carbons resonate in the region of 198.9-201.6 ppm, the signal of the cyano group carbon appears at 111.1-111.9 ppm. In the IR spectra of the products $4 a-e$, the $C=N$ and $\mathrm{C}=\mathrm{O}$ absorption bands are observed at 2229-2221 and $1724-1714 \mathrm{~cm}^{-1}$, respectively. The valence vibrations of the double bond in the $3(2 \mathrm{H})$-furanone alkenonitrile fragment are in the region of $1646-1552 \mathrm{~cm}^{-}$ 1 .

The assembly of the both functionalized 2,3-dihydrofurans 3 and 3(2H)-furanones 4 involves the intermediate keto esters $\mathbf{5}$ that results from the following cascade: nucleophilic addition of carboxylic acids $\mathbf{2}$ to the triple bond of cyanopropargyl alcohol $\mathbf{1}$ and intramolecular transesterification in the adducts $\mathbf{A}$ to give keto esters 5 (Scheme 2). Then two competitive reactions take place: (i) intramolecular cyclization of intermediates 5 to $3(2 \mathrm{H})$-furanones $\mathbf{4}$ or (ii) the nucleophilic attack of carbanions $\mathbf{B}$ at the triple bond of the second molecule of cyanopropargyl alcohol $\mathbf{1}$ and the carbanions $\mathbf{C}$ finally cyclize to 2,3-dihydrofurans $\mathbf{3}$ (Scheme 2).
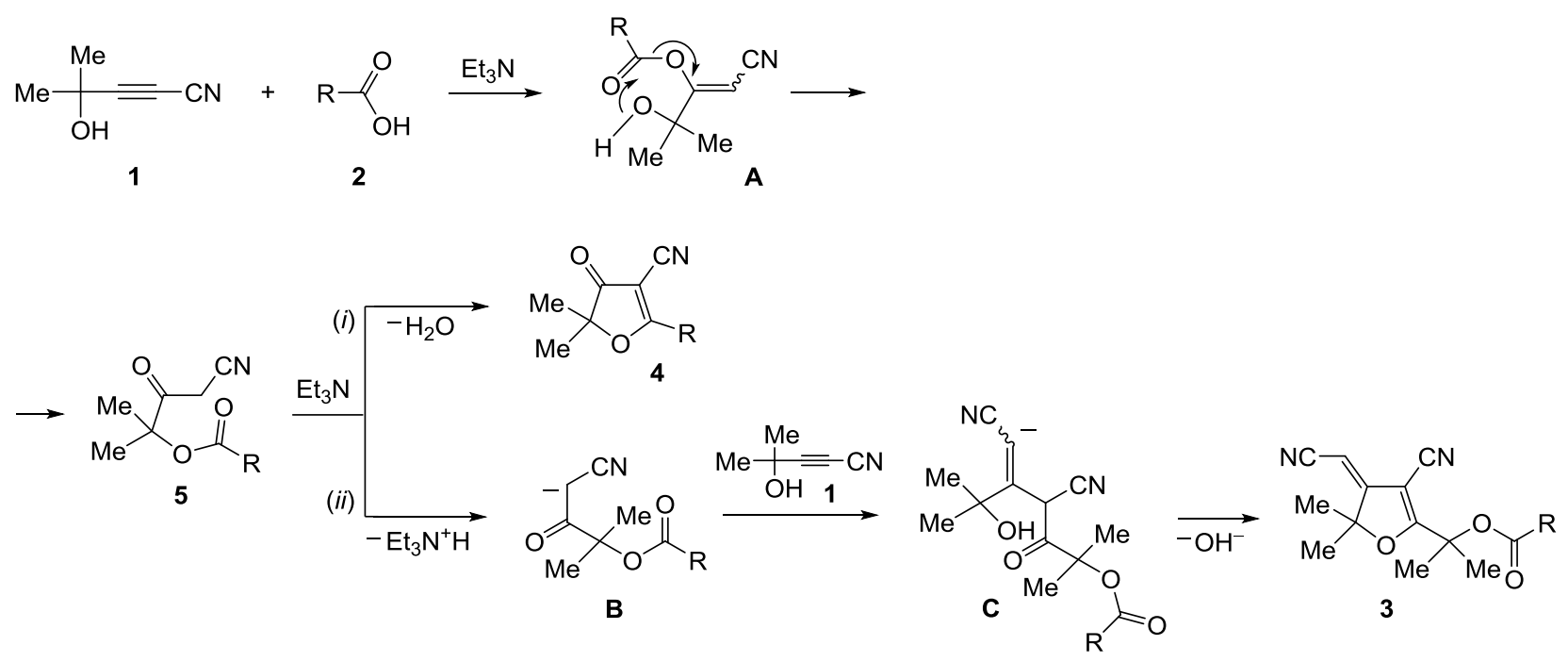

Scheme 2. Possible mechanism of 2,3-dihydrofurans 3 and 3(2H)-furanones 4 formation.

The milder conditions of the assembly of cyanopropargyl alcohol with aliphatic acids in contrast to analogous reactions with heteroaromatic carboxylic acids ${ }^{37-40}$ can be understood in terms of a higher nucleophilicity of the anions of aliphatic carboxylic acids that should make the first stage of the cascade faster. On the other hand, the aliphatic substituents in the carboxylic group decrease the electrophilicity of the carbanion moiety and hence should slow down the intramolecular cyclization of the intermediates $\mathbf{5}$ (i). This likely allows the intermolecular transformation of the carbanions $\mathbf{B}$ (ii) to be more competitive and therefore to increase the relative content of 2,3-dihydrofurans $\mathbf{3}$ in the reaction mixture. The observed stereoselectivity of 2:1 assembly of 4-cyano-[(Z)-3-cyanomethylene]-2,3-dihydrofurans 3 is due to preference for the trans- 
configuration of the carbanions $\mathbf{C}$ resulted from the nucleophilic attack at the triple bond of cyanopropargyl alcohol 1.

\section{Conclusions}

In conclusion, aliphatic carboxylic acids have been successfully involved under mild conditions into one-pot metal-free cascade assembly with available tertiary cyanopropargyl alcohol to afford functionalized 2,3dihydrofurans and $3(2 \mathrm{H})$-furanones in good total yields. The observed differences of the synthesis are due to a higher nucleophilicity of aliphatic carboxylate anions and a lower electrophilicity of the carbonyl group of the aliphatic acids participating in intramolecular 1:1 assembly of 3(2H)-furanone scaffold. These effects and their competition favor the intermolecular 2:1 assembly to 2,3-dihydrofurans and hinder $1: 1$ assembly to $3(2 H)$ furanones. The intermediate cyano substituted keto esters, common for the both directions, have been shown to be easily isolable in synthetically acceptable yields thus representing a new highly reactive family of synthetic building blocks. The results substantially extend the substrate scope of the synthesis and broaden structural diversity of the functionalized dihydrofurans, particularly having in mind availability and abundance of aliphatic carboxylic acids as the fundamental class of organic compounds.

\section{Experimental Section}

General. ${ }^{1} \mathrm{H}$ and ${ }^{13} \mathrm{C}$ NMR spectra were recorded on a Bruker DPX-400 spectrometer (400.1 and $100.6 \mathrm{MHz}$, respectively) in $\mathrm{CDCl}_{3}$ using hexamethyldisiloxane as internal references at $20-25{ }^{\circ} \mathrm{C}$. For labeling of NMR assignments, see Figure 1.

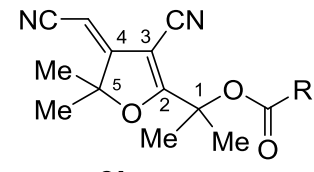

3b-e

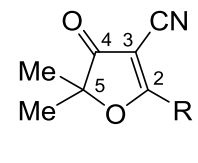

4b-e

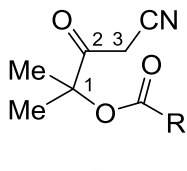

5b-e

Figure 1. Labeling of hydrogen and carbon atoms in compounds $3 \mathbf{b}-\mathbf{e}, \mathbf{4 b - e ,} \mathbf{5 b}-\mathbf{e}$.

IR spectra were measured on a Bruker Vertex-70 instrument in thin films or $\mathrm{KBr}$ pellets. Microanalyses were performed on a Flash 2000 elemental analyzer. Melting points were determined using a Kofler micro hot stage. Mass spectra were recorded on a GCMS-QP5050A spectrometer made by Shimadzu Company. Chromatographic column parameters were as follows: $\mathrm{SPB}^{\mathrm{TM}}-5$, length $60 \mathrm{~m}$, internal diameter $0.25 \mathrm{~mm}$, thickness of stationary phase film $0.25 \mu \mathrm{m}$; injector temperature $250{ }^{\circ} \mathrm{C}$, gas carrier - helium, flow rate 0.7 $\mathrm{mL} / \mathrm{min}$; detector temperature $250^{\circ} \mathrm{C}$; mass analyzer: quadrupole, electron ionization, electron energy: $70 \mathrm{eV}$, ion source temperature $200{ }^{\circ} \mathrm{C}$; mass range 34-650 Da. The solvent was MeCN (spectroscopic grade) from Scientific Production Company "Cryochrom" (St. Petersburg, Russia). Column chromatography was performed on silica gel 60 (70-230 mesh, particle size 0.063-0.200 nm, Merck). Acetic, butyric, pentanoic, 2methylpropanoic and 3-methylbutanoic acids 2a-e are commercial reagents. Cyanopropargyl alcohol 1 were prepared according to a published method. ${ }^{43}$ Commercially available starting materials were used without further purification. 


\section{Reaction of 4-hydroxy-4-methyl-2-pentynenitrile (1) with aliphatic carboxylic acids 2.}

(a) Triethylamine (101 mg, $1 \mathrm{mmol}$ ) was added dropwise over $1 \mathrm{~min}$ to a stirred solution of 4-hydroxy-4methylpent-2-ynenitrile (1; $109 \mathrm{mg}, 1 \mathrm{mmol}$ ) and acetic acid (2a; $60 \mathrm{mg}, 1 \mathrm{mmol})$ in MeCN (5 mL). The reaction mixture was stirred at $20-25^{\circ} \mathrm{C}$ for $48 \mathrm{~h}$, then concentrated and the obtained residue was passed through silica gel $\left(3 \mathrm{~cm}^{3}, \mathrm{Et}_{2} \mathrm{O}\right)$ to give (Z)-2-[3-cyano-4-(cyanomethylene)-5,5-dimethyl-4,5-dihydrofuran-2-yl]propan-2-yl acetate (3a; 14 mg, 11\%; colorless oil) and 2,5,5-trimethyl-4-oxo-4,5-dihydro-3-furancarbonitrile (4a; $131 \mathrm{mg}$, $87 \%$; colorless crystals; $\mathrm{mp} 78-80^{\circ} \mathrm{C}$ ). Analyzing data for $3 \mathrm{a}$ and $4 \mathrm{a}$ were published in literature. ${ }^{40}$

(b) Triethylamine(101 mg, $1 \mathrm{mmol}$ ) was added dropwise over $1 \mathrm{~min}$ to a stirred solution of 1 (218 mg, $2 \mathrm{mmol})$ and $2 \mathrm{a}(60 \mathrm{mg}, 1 \mathrm{mmol})$. The reaction mixture was stirred at $20-25{ }^{\circ} \mathrm{C}$ for $48 \mathrm{~h}$, then concentrated and the obtained residue was purified by column chromatography $\left(1.0 \times 50 \mathrm{~cm}, \mathrm{SiO}_{2}, \mathrm{Et}_{2} \mathrm{O}-\mathrm{C}_{6} \mathrm{H}_{14}, 1: 1\right)$ to give $2,3-$ dihydrofuran 3 a (98 $\mathrm{mg}, 38 \%$ ) and 3(2H)-furanone 4 a (30 mg, 20\%).

(c) Triethylamine (101 mg, $1 \mathrm{mmol}$ ) was added dropwise over $1 \mathrm{~min}$ to a stirred solution of 1 (327 mg, $3 \mathrm{mmol}$ ) and $2 \mathrm{a}(60 \mathrm{mg}, 1 \mathrm{mmol})$. The reaction mixture was stirred at $20-25{ }^{\circ} \mathrm{C}$ for $48 \mathrm{~h}$, then concentrated and the obtained residue was purified by column chromatography $\left(1.0 \times 50 \mathrm{~cm}, \mathrm{SiO}_{2}, \mathrm{Et}_{2} \mathrm{O}-\mathrm{C}_{6} \mathrm{H}_{14}, 1: 1\right)$ to give mixture $(178 \mathrm{mg})$ of the 2,3-dihydrofuran 3a and 4-hydroxy-4-methyl-2-pentynenitrile (1), and 3(2H)-furanone 4a (11 $\mathrm{mg}, 9 \%)$. The mixture of the $3 \mathrm{a}$ and 1 was additionally purified by column chromatography $\left(1.0 \times 50 \mathrm{~cm}, \mathrm{SiO}_{2}\right.$, $\left.\mathrm{CHCl}_{3}-\mathrm{C}_{6} \mathrm{H}_{14}-\mathrm{Et}_{2} \mathrm{O}, 20: 10: 1\right)$ to give the desired product $3 \mathrm{a}$ (109 mg, 42\%) and initial 4-hydroxy-4-methylpent2-ynenitrile (1; $69 \mathrm{mg}$, conversion 79\%).

(d) Triethylamine (202 mg, $2 \mathrm{mmol}$ ) was added dropwise over $1 \mathrm{~min}$ to a stirred solution of 4-hydroxy-4methylpent-2-ynenitrile (1; $131 \mathrm{mg}, 1.2 \mathrm{mmol}$ ) and pentanoic acid (2c; $102 \mathrm{mg}, 1 \mathrm{mmol}$ ) in MeCN (5 mL). The reaction mixture was stirred at $20-25{ }^{\circ} \mathrm{C}$ for $48 \mathrm{~h}$, then concentrated and the obtained residue was purified by column chromatography $\left(1.0 \times 50 \mathrm{~cm}, \mathrm{SiO}_{2}, \mathrm{Et}_{2} \mathrm{O}-\mathrm{C}_{6} \mathrm{H}_{14}, 1: 1\right)$ to give 2,3-dihydrofuran $3 \mathrm{c}(14 \mathrm{mg}, 9 \%), 3(2 \mathrm{H})$ furanone $4 \mathrm{c}$ (193 $\mathrm{mg}, 63 \%)$ and keto ester $5 \mathrm{c}$ ( $29 \mathrm{mg}, 90 \%$ pure as determined by ${ }^{1} \mathrm{H}$ NMR, 12\% yield).

(e) Triethylamine (53 mg, $0.5 \mathrm{mmol}$ ) was added dropwise over $1 \mathrm{~min}$ to a stirred solution of 4-hydroxy-4methylpent-2-ynenitrile (1; $56 \mathrm{mg}, 0.5 \mathrm{mmol}$ ) and 3-methylbutanoic acid (2e; $52 \mathrm{mg}, 0.5 \mathrm{mmol})$ in MeCN (2.5 $\mathrm{mL}$ ). The reaction mixture was stirred at $80{ }^{\circ} \mathrm{C}$ for $9.5 \mathrm{~h}$, then concentrated and the obtained residue was purified by column chromatography $\left(1.0 \times 30 \mathrm{~cm}, \mathrm{SiO}_{2}, \mathrm{Et}_{2} \mathrm{O}-\mathrm{C}_{6} \mathrm{H}_{14}, 1: 1\right)$ to give $3(2 \mathrm{H})$-furanone $4 \mathrm{e}(55 \mathrm{mg}$, $57 \%$ ) and keto ester 5 e ( $6 \mathrm{mg}, 70 \%$ pure as determined by ${ }^{1} \mathrm{H} \mathrm{NMR}, 4 \%$ yield).

Reaction of 4-hydroxy-4-methyl-2-pentynenitrile (1) with butyric acid (2b). Following the procedure (a) using 1 (131 mg, $1.2 \mathrm{mmol}), 2 \mathbf{b}(88 \mathrm{mg}, 1 \mathrm{mmol})$, and $\mathrm{Et}_{3} \mathrm{~N}(101 \mathrm{mg}, 1 \mathrm{mmol})$ in $\mathrm{MeCN}(5 \mathrm{~mL})$ a mixture of products 3b, $\mathbf{4 b}$ and $\mathbf{5 b}$ was obtained. Purification: column chromatography $\left(1.0 \times 40 \mathrm{~cm}, \mathrm{SiO}_{2}, \mathrm{Et}_{2} \mathrm{O}-\mathrm{C}_{6} \mathrm{H}_{14}, 1: 1\right)$; this gave $\mathbf{3 b}$ (51 mg, 18\%), 4b (110 mg, 61\%) and $\mathbf{5 b}$ (38 mg, 90\% pure as determined by ${ }^{1} \mathrm{H}$ NMR, $17 \%$ yield).

Following the procedure (b) using $1(218 \mathrm{mg}, 2 \mathrm{mmol}), \mathbf{2 b}(88 \mathrm{mg}, 1 \mathrm{mmol})$, and $\mathrm{Et}_{3} \mathrm{~N}(101 \mathrm{mg}, 1 \mathrm{mmol}) \mathrm{a}$ mixture of products $\mathbf{3 b}, \mathbf{4 b}$ and $\mathbf{5 b}$ was obtained. Subsequent purification gave $\mathbf{3 b}(169 \mathrm{mg}, 59 \%), \mathbf{4 b}(26 \mathrm{mg}$, $15 \%$ ) and $\mathbf{5 b}$ (40 $\mathrm{mg}, 85 \%$ pure as determined by ${ }^{1} \mathrm{H}$ NMR, $17 \%$ yield).

(Z)-2-[3-Cyano-4-(cyanomethylene)-5,5-dimethyl-4,5-dihydrofuran-2-yl]propan-2-yl butyrate (3b). Colorless oil. Z/E 85/15; IR (film): 3094, 3066 (C=CH), 2994, 2976, 2937 (CH), 2223 and 2211 (C=N), 1713 (C=O), 1645, 1593, $1563(\mathrm{C}=\mathrm{C}) \mathrm{cm}^{-1} ;{ }^{1} \mathrm{H}$ NMR $\left(400.1 \mathrm{MHz} \mathrm{CDCl}_{3}\right)$ : Z-isomer $\delta=5.09(\mathrm{~s}, 1 \mathrm{H},=\mathrm{CHCN}), 2.35(\mathrm{~m}, 2 \mathrm{H}$, $\left.\mathrm{CH}_{3} \mathrm{CH}_{2} \mathrm{CH}_{2}\right), 1.71\left[\mathrm{~s}, 6 \mathrm{H},\left(\mathrm{CH}_{3}\right)_{2} \mathrm{C}-1\right], 1.68\left[\mathrm{~s}, 6 \mathrm{H},\left(\mathrm{CH}_{3}\right)_{2} \mathrm{C}-5\right], 1.63\left(\mathrm{~m}, 1 \mathrm{H}, \mathrm{CH}_{3} \mathrm{C}_{2} \mathrm{CH}_{2}\right), 0.94\left(\mathrm{t}, 3 \mathrm{H}, \mathrm{C}_{3} \mathrm{CH}_{2} \mathrm{CH}_{2}\right.$, J $7.4 \mathrm{~Hz}$ ); E-isomer $\delta=5.05(\mathrm{~s}, 1 \mathrm{H},=\mathrm{CHCN}), 2.55\left(\mathrm{~m}, 2 \mathrm{H}, \mathrm{CH}_{3} \mathrm{CH}_{2} \mathrm{CH}_{2}\right), 1.71\left[\mathrm{~s}, 6 \mathrm{H},\left(\mathrm{CH}_{3}\right)_{2} \mathrm{C}-1\right], 1.68[\mathrm{~s}, 6 \mathrm{H}$, $\left.\left(\mathrm{CH}_{3}\right)_{2} \mathrm{C}-5\right], 1.63\left(\mathrm{~m}, 1 \mathrm{H}, \mathrm{CH}_{3} \mathrm{CH}_{2} \mathrm{CH}_{2}\right), 0.99\left(\mathrm{t}, 3 \mathrm{H}, \mathrm{CH}_{3} \mathrm{CH}_{2} \mathrm{CH}_{2}, J 7.4 \mathrm{~Hz}\right) ;{ }^{13} \mathrm{C} \mathrm{NMR}\left(100.6 \mathrm{MHz}, \mathrm{CDCl}_{3}\right)$ : Z-isomer

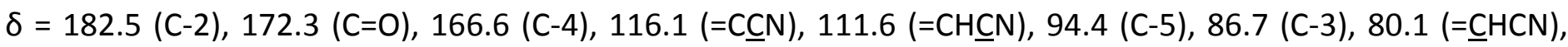
75.9 (C-1), $35.7\left(\mathrm{CH}_{3} \mathrm{CH}_{2} \underline{\mathrm{CH}_{2}}\right), 25.1\left[\left(\mathrm{CH}_{3}\right)_{2} \mathrm{C}-1\right], 24.5\left[\left(\mathrm{CH}_{3}\right)_{2} \mathrm{C}-5\right], 18.1\left(\mathrm{CH}_{3} \underline{\mathrm{CH}}_{2} \mathrm{CH}_{2}\right), 13.4\left(\underline{\mathrm{CH}}_{3} \mathrm{CH}_{2} \mathrm{CH}_{2}\right)$; E-isomer 


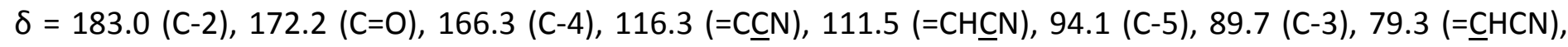
77.2 (C-1), $30.8\left(\mathrm{CH}_{3} \mathrm{CH}_{2} \underline{\mathrm{C}} \mathrm{H}_{2}\right), 25.1\left[\left(\mathrm{CH}_{3}\right)_{2} \mathrm{C}-1\right]$, 24.7 [ $\left.\left(\mathrm{CH}_{3}\right)_{2} \mathrm{C}-5\right], 19.7\left(\mathrm{CH}_{3} \mathrm{CH}_{2} \mathrm{CH}_{2}\right), 13.3\left(\underline{\mathrm{C}} \mathrm{CH}_{3} \mathrm{CH}_{2} \mathrm{CH}\right)$; MS (EI): $m / z(\%)=288(21)[\mathrm{M}]^{+}, 201$ (11), 175 (27), 71 (100), 43 (93), 41 (27); Anal. Calcd for $\mathrm{C}_{16} \mathrm{H}_{20} \mathrm{~N}_{2} \mathrm{O}_{3}$ (288.34): C $66.65 ; \mathrm{H} 6.99 ; \mathrm{N} 9.72$. Found: C, 66.91; H, 6.72; N, 9.50.

5,5-Dimethyl-4-oxo-2-propyl-4,5-dihydro-3-furancarbonitrile (4b). White powder; mp 84-86 ${ }^{\circ} \mathrm{C} ; \mathrm{IR}(\mathrm{KBr})$ : 3092, $3068(\mathrm{C}=\mathrm{CH})$, 2995, 2973, $2934(\mathrm{CH}), 2221(\mathrm{C}=\mathrm{N}), 1714(\mathrm{C}=\mathrm{O}), 1644,1595,1562(\mathrm{C}=\mathrm{C}) \mathrm{cm}^{-1} ;{ }^{1} \mathrm{H} \mathrm{NMR}$ $\left(400.1 \mathrm{MHz}, \mathrm{CDCl}_{3}\right): \delta=2.34\left(\mathrm{~m}, 2 \mathrm{H}, \mathrm{CH}_{3} \mathrm{CH}_{2} \underline{\mathrm{C}}_{2}\right), 1.80\left(\mathrm{~m}, 2 \mathrm{H}, \mathrm{CH}_{3} \underline{\mathrm{C}}_{2}\right), 1.45\left(\mathrm{~s}, 6 \mathrm{H}, 2 \mathrm{CH}_{3}\right), 1.02(\mathrm{t}, 3 \mathrm{H}$, $\mathrm{CH}_{3} \mathrm{CH}_{2}, J 7.3 \mathrm{~Hz}$ ); ${ }^{13} \mathrm{C}$ NMR (100.6 MHz, CDCl 3 ): $\delta=198.9$ (C=O), 197.6 (C-2), 111.9 (CN), 94.0 (C-5), 91.7 (C-3), $32.5\left(\mathrm{CH}_{3} \mathrm{CH}_{2} \underline{\mathrm{CH}}_{2}\right), 24.7\left(2 \mathrm{CH}_{3}\right), 19.6\left(\mathrm{CH}_{3} \mathrm{CH}_{2}\right), 13.6\left(\underline{\mathrm{CH}}_{3} \mathrm{CH}_{2}\right) ; \mathrm{MS}(\mathrm{EI}): \mathrm{m} / z(\%)=179(17), 177(12)[\mathrm{M}-2 \mathrm{H}]^{+}, 71$ (100), 43 (36), 41 (16); Anal. Calcd for $\mathrm{C}_{10} \mathrm{H}_{13} \mathrm{NO}_{2}$ (179.22): C 67.02; H 7.31; N 7.82. Found: C 67.27; H 7.24; N 8.01 .

3-Cyano-1,1-dimethyl-2-oxopropyl butyrate (5b). ${ }^{1} \mathrm{H} \mathrm{NMR}\left(400.1 \mathrm{MHz}, \mathrm{CDCl}_{3}\right): \delta=3.54\left(\mathrm{~s}, 2 \mathrm{H}, \mathrm{CH}_{2}-\mathrm{CN}\right), 2.33$ (m, $\left.2 \mathrm{H}, \mathrm{CH}_{3} \mathrm{CH}_{2} \mathrm{CH}_{2}\right), 1.65\left(\mathrm{~m}, 2 \mathrm{H}, \mathrm{CH}_{3} \mathrm{CH}_{2} \mathrm{CH}_{2}\right), 1.51\left(\mathrm{~s}, 6 \mathrm{H}, 2 \mathrm{CH}_{3}\right), 0.96\left(\mathrm{t}, 3 \mathrm{H}, \mathrm{CH}_{3} \mathrm{CH}_{2} \mathrm{CH}_{2}, J 7.4 \mathrm{~Hz}\right) ;{ }^{13} \mathrm{CNMR}^{\mathrm{N}}$ $\left(100.6 \mathrm{MHz}, \mathrm{CDCl}_{3}\right): \delta=196.4(\mathrm{C}=\mathrm{O}), 173.5\left(\mathrm{CO}_{2}\right), 113.6(\mathrm{CN}), 82.9(\mathrm{C}-1), 35.9\left(\mathrm{CH}_{3} \mathrm{CH}_{2} \mathrm{CH}_{2}\right), 26.6\left(\underline{\mathrm{C}} \mathrm{H}_{2} \mathrm{CN}\right), 23.2$ $\left(2 \mathrm{CH}_{3}\right), 18.2\left(\mathrm{CH}_{3} \underline{\mathrm{CH}}_{2} \mathrm{CH}_{2}\right), 13.5\left(\mathrm{CH}_{3} \mathrm{CH}_{2} \mathrm{CH}_{2}\right) ; \mathrm{MS}(\mathrm{EI}): \mathrm{m} / z(\%)=195$ (16) [M-2H], 138 (21), 137 (19), 124 (10), 110 (11), 70 (35), 69 (13), 66 (16), 59 (100), 43 (92), 42 (14), 41 (26), 39 (17).

Reaction of 4-hydroxy-4-methyl-2-pentynenitrile (1) with pentanoic acid (2c). Following the procedure (a) using 1 (131 mg, $1.2 \mathrm{mmol}), 2 \mathrm{c}(102 \mathrm{mg}, 1 \mathrm{mmol})$, and $\mathrm{Et}_{3} \mathrm{~N}(101 \mathrm{mg}, 1 \mathrm{mmol})$ in MeCN (5 mL) a mixture of products 3c, 4c and 5c was obtained. Purification: column chromatography $\left(1.0 \times 40 \mathrm{~cm}, \mathrm{SiO}_{2}, \mathrm{Et}_{2} \mathrm{O}-\mathrm{C}_{6} \mathrm{H}_{14}\right.$, 1:1); this gave $3 \mathrm{c}(15 \mathrm{mg}, 10 \%), 4 \mathrm{c}(115 \mathrm{mg}, 60 \%)$ and $5 \mathrm{c}\left(36 \mathrm{mg}, 80 \%\right.$ pure as determined by ${ }^{1} \mathrm{H}$ NMR, $14 \%$ yield).

Following the procedure (b) using 1 (218 mg, $2 \mathrm{mmol}), 2 \mathrm{c}(102 \mathrm{mg}, 1 \mathrm{mmol})$, and Et ${ }_{3} \mathrm{~N}(101 \mathrm{mg}, 1 \mathrm{mmol}) \mathrm{a}$ mixture of products 3c, 4c and 5c was obtained. Subsequent purification gave 3c (120 mg, 40\%), 4c (29, mg 15\%) and $5 \mathrm{c}$ (16 $\mathrm{mg}, 75 \%$ pure as determined by ${ }^{1} \mathrm{H} N M R, 6 \%$ yield).

(Z)-2-[3-Cyano-4-(cyanomethylene)-5,5-dimethyl-4,5-dihydrofuran-2-yl]propan-2-yl pentanoate (3c). Light yellow oil. Z/E 90/10; IR (film): 3047 (C=C), 2986, 2960, 2938, 2872 (CH), 2218 (CN), 1741 (CO), 1623, 1584 $(\mathrm{C}=\mathrm{C}) \mathrm{cm}^{-1} ;{ }^{1} \mathrm{H}$ NMR $\left(400.1 \mathrm{MHz}, \mathrm{CDCl}_{3}\right)$ : Z- isomer $\delta=5.09(\mathrm{~s}, 1 \mathrm{H},=\mathrm{CHCN}), 2.36\left(\mathrm{~m}, 2 \mathrm{H}, \mathrm{CH}_{3} \mathrm{CH}_{2} \mathrm{CH}_{2} \mathrm{C}_{2}\right), 1.71$ [s, $\left.6 \mathrm{H},\left(\mathrm{CH}_{3}\right)_{2} \mathrm{C}-1\right], 1.68\left[\mathrm{~s}, 6 \mathrm{H},\left(\mathrm{CH}_{3}\right)_{2} \mathrm{C}-5\right], 1.59\left(\mathrm{~m}, 2 \mathrm{H}, \mathrm{CH}_{3} \mathrm{C}_{2} \mathrm{CH}_{2} \mathrm{CH}_{2}\right), 1.35\left(\mathrm{~m}, 2 \mathrm{H}, \mathrm{CH}_{3} \mathrm{CH}_{2} \mathrm{CH}_{2} \mathrm{CH}_{2}\right), 0.92(\mathrm{t}$, $3 \mathrm{H}, \mathrm{CH}_{3} \mathrm{CH}_{2} \mathrm{CH}_{2} \mathrm{CH}_{2}, J 7.3 \mathrm{~Hz}$ ); $\mathrm{E}$ - isomer $\delta=5.04(\mathrm{~s}, 1 \mathrm{H},=\mathrm{CHCN}), 2.57\left(\mathrm{~m}, 2 \mathrm{H}, \mathrm{CH}_{3} \mathrm{CH}_{2} \mathrm{CH}_{2} \mathrm{C}_{2}\right), 1.71[\mathrm{~s}, 6 \mathrm{H}$, $\left.\left(\mathrm{CH}_{3}\right)_{2} \mathrm{C}-1\right], 1.68\left[\mathrm{~s}, 6 \mathrm{H},\left(\mathrm{CH}_{3}\right)_{2} \mathrm{C}-5\right], 1.59\left(\mathrm{~m}, 2 \mathrm{H}, \mathrm{CH}_{3} \underline{C}_{2} \mathrm{CH}_{2} \mathrm{CH}_{2}\right), 1.35\left(\mathrm{~m}, 2 \mathrm{H}, \mathrm{CH}_{3} \mathrm{CH}_{2} \mathrm{CH}_{2} \mathrm{CH}_{2}\right), 0.94(\mathrm{t}, 3 \mathrm{H}$, $\mathrm{CH}_{3} \mathrm{CH}_{2} \mathrm{CH}_{2} \mathrm{CH}_{2}, J 7.3 \mathrm{~Hz}$ ); ${ }^{13} \mathrm{C}$ NMR $\left(100.6 \mathrm{MHz}, \mathrm{CDCl}_{3}\right)$ : Z- isomer $\delta=182.6(\mathrm{C}-2), 172.5(\mathrm{C}=0), 166.6(\mathrm{C}-4)$, 116.2 (=CN), 111.6 (=CHCN), 94.4 (C-5), 86.6 (C-3), 80.1 (= $\underline{\mathrm{CHCN}}$ ), 75.8 (C-1), $33.6\left(\mathrm{CH}_{3} \mathrm{CH}_{2} \mathrm{CH}_{2} \underline{\mathrm{CH}_{2}}\right), 26.7$ $\left(\mathrm{CH}_{3} \mathrm{CH}_{2} \underline{\mathrm{CH}}_{2} \mathrm{CH}_{2}\right), 25.1\left[(\underline{\mathrm{CH}})_{2} \mathrm{C}-1\right]$, $24.5\left[\left(\mathrm{CH}_{3}\right)_{2} \mathrm{C}-5\right], 22.0\left(\mathrm{CH}_{3} \underline{\mathrm{C}}_{2} \mathrm{CH}_{2} \mathrm{CH}_{2}\right), 13.6\left(\underline{\mathrm{CH}}_{3} \mathrm{CH}_{2} \mathrm{CH}_{2} \mathrm{CH}_{2}\right) ; \mathrm{E}-$ isomer $\delta=$

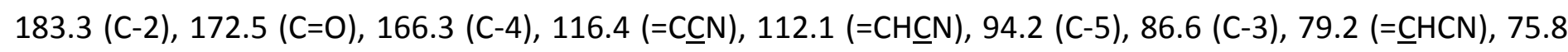
(C-1), $28.7\left(\mathrm{CH}_{3} \mathrm{CH}_{2} \mathrm{CH}_{2} \underline{\mathrm{CH}_{2}}\right), 28.1\left(\mathrm{CH}_{3} \mathrm{CH}_{2} \mathrm{CH}_{2} \mathrm{CH}_{2}\right), 24.7\left[\left(\underline{\mathrm{CH}_{3}}\right)_{2} \mathrm{C}-1\right], 24.5\left[\left(\underline{\mathrm{CH}}_{3}\right)_{2} \mathrm{C}-5\right], 22.0\left(\mathrm{CH}_{3} \mathrm{C}_{2} \mathrm{CH}_{2} \mathrm{CH}_{2}\right)$, $13.5\left(\mathrm{CH}_{3} \mathrm{CH}_{2} \mathrm{CH}_{2} \mathrm{CH}_{2}\right)$; MS (EI): $m / z(\%)=302$ (54) [M] ${ }^{+}, 203$ (12), 202 (27), 201 (26), 200 (15), 175 (34), 85 (100), 69 (11), 57 (92), 43 (35), 41 (51), 39 (11); Anal. Calcd for $\mathrm{C}_{17} \mathrm{H}_{22} \mathrm{~N}_{2} \mathrm{O}_{3}$ (302.37): C, 67.53; H, 7.33; N, 9.26. Found: C, 67.91; H, 7.72; N, 9.50.

2-Butyl-5,5-dimethyl-4-oxo-4,5-dihydro-3-furancarbonitrile (4c). Light yellow oil; IR (film): 2963, 2937, 2873 (CH), 2229 (CN), 1723 (C=O), $1587(\mathrm{C}=\mathrm{C}) \mathrm{cm}^{-1} ;{ }^{1} \mathrm{H} \mathrm{NMR}\left(400.1 \mathrm{MHz}, \mathrm{CDCl}_{3}\right): \delta=2.76\left(\mathrm{~m}, 2 \mathrm{H}, \mathrm{CH}_{3} \mathrm{CH}_{2} \mathrm{CH}_{2} \mathrm{CH}_{2}\right)$, $1.73\left(\mathrm{~m}, 2 \mathrm{H}, \mathrm{CH}_{3} \mathrm{CH}_{2} \mathrm{CH}_{2} \mathrm{CH}_{2}\right), 1.44\left(\mathrm{~s}, 6 \mathrm{H}, 2 \mathrm{CH}_{3}\right), 1.41\left(\mathrm{~m}, 2 \mathrm{H}, \mathrm{CH}_{3} \mathrm{CH}_{2} \mathrm{CH}_{2} \mathrm{CH}_{2}\right), 0.96\left(\mathrm{t}, 3 \mathrm{H}_{,} \mathrm{CH}_{3} \mathrm{CH}_{2} \mathrm{CH}_{2} \mathrm{CH}_{2}, J\right.$ $7.3 \mathrm{~Hz}$ ); ${ }^{13} \mathrm{C}$ NMR (100.6 MHz, CDCl 3 ): $\delta=199.0$ (C=O), 197.9 (C-2), 111.3 (CN), 91.8 (C-5), 91.1 (C-3), 30.6 $\left(\mathrm{CH}_{3} \mathrm{CH}_{2} \underline{\mathrm{CH}_{2}} \mathrm{CH}_{2}\right), 27.8\left(\mathrm{CH}_{3} \mathrm{CH}_{2} \mathrm{CH}_{2} \underline{\mathrm{CH}_{2}}\right), 22.6\left(2 \mathrm{CH}_{3}\right), 22.1\left(\mathrm{CH}_{3} \underline{C H}_{2} \mathrm{CH}_{2} \mathrm{CH}_{2}\right), 13.5\left(\underline{C H}_{3} \mathrm{CH}_{2} \mathrm{CH}_{2} \mathrm{CH}_{2}\right) ; \mathrm{MS}(\mathrm{EI}): m / z$ 
$(\%)=193(44)[\mathrm{M}]^{+}, 192$ (11), 164 (20), 151 (22), 136 (26), 123 (65), 108 (14), 107 (15), 106 (16), 93 (26), 69 (16), 65 (11), 59 (21), 58 (25), 57 (11), 43 (100), 42 (13), 42 (13), 41 (51), 39 (27); Anal. Calcd for $\mathrm{C}_{11} \mathrm{H}_{15} \mathrm{NO}_{2}$ (193.24): C, 68.37; H, 7.82; N, 7.25. Found: C, 68.16; H, 8.14; N, 7.51.

3-Cyano-1,1-dimethyl-2-oxopropyl pentanoate (5c). ${ }^{1} \mathrm{H} \mathrm{NMR}\left(400.1 \mathrm{MHz}, \mathrm{CDCl}_{3}\right): \delta=3.54\left(\mathrm{~s}, 2 \mathrm{H}, \mathrm{CH}_{2} \mathrm{CN}\right), 2.35$ (m, $\left.2 \mathrm{H}, \mathrm{CH}_{3} \mathrm{CH}_{2} \mathrm{CH}_{2} \mathrm{C}_{2}\right), 1.60\left(\mathrm{~m}, 2 \mathrm{H}, \mathrm{CH}_{3} \mathrm{CH}_{2} \mathrm{C}_{2} \mathrm{CH}_{2}\right), 1.51\left(\mathrm{~s}, 6 \mathrm{H}, 2 \mathrm{CH}_{3}\right), 1.34\left(\mathrm{~m}, 2 \mathrm{H}, \mathrm{CH}_{3} \mathrm{CH}_{2} \mathrm{CH}_{2} \mathrm{CH}_{2}\right), 0.92$ (t, $3 \mathrm{H}, \mathrm{CH}_{3} \mathrm{CH}_{2} \mathrm{CH}_{2} \mathrm{CH}_{2}, J 7.3 \mathrm{~Hz}$ ); ${ }^{13} \mathrm{C} \mathrm{NMR}\left(100.6 \mathrm{MHz}, \mathrm{CDCl}_{3}\right): \delta=196.4(\mathrm{C}=\mathrm{O}), 173.8\left(\mathrm{CO}_{2}\right), 113.6$ (CN), 82.8 (C1), $33.8\left(\mathrm{CH}_{3} \mathrm{CH}_{2} \mathrm{CH}_{2} \underline{\mathrm{CH}}_{2}\right), 26.7\left(\mathrm{CH}_{3} \mathrm{CH}_{2} \mathrm{CH}_{2} \mathrm{CH}_{2}\right), 26.6\left(\mathrm{CH}_{2} \mathrm{CN}\right), 23.1\left(2 \mathrm{CH}_{3}\right), 22.1\left(\mathrm{CH}_{3} \mathrm{CH}_{2} \mathrm{CH}_{2} \mathrm{CH}_{2}\right), 13.6$ $\left(\mathrm{CH}_{3} \mathrm{CH}_{2} \mathrm{CH}_{2} \mathrm{CH}_{2}\right)$; $\mathrm{MS}(\mathrm{EI}): \mathrm{m} / z$ (\%) = 193 (10) $\left[\mathrm{M}-\mathrm{H}_{2} \mathrm{O}\right]^{+}, 136$ (12), 123 (25), 93 (15), 59 (17), 58 (19), 43 (100), 42 (12), 41 (43), 39 (26).

\section{Reaction of 4-hydroxy-4-methyl-2-pentynenitrile (1) with 2-methylpropanoic acid (2d)}

Following the procedure (a) using 1 (131 mg, $1.2 \mathrm{mmol}), 2 \mathbf{d}(88 \mathrm{mg}, 1 \mathrm{mmol})$, and $\mathrm{Et}_{3} \mathrm{~N}(101 \mathrm{mg}, 1 \mathrm{mmol}) \mathrm{in}$ $\operatorname{MeCN}(5 \mathrm{~mL})$ a mixture of products $\mathbf{3 d}, \mathbf{4 d}$ and $\mathbf{5 d}$ was obtained. Purification: column chromatography $(1.0 \times$ $\left.40 \mathrm{~cm}, \mathrm{SiO}_{2}, \mathrm{Et}_{2} \mathrm{O}-\mathrm{C}_{6} \mathrm{H}_{14}, 1: 1\right)$; this gave $\mathbf{3 d}(12 \mathrm{mg}, 8 \%), \mathbf{4 d}(82 \mathrm{mg}, 46 \%)$ and $\mathbf{5 d}(77 \mathrm{mg}, 95 \%$ pure as determined by ${ }^{1} \mathrm{H}$ NMR, $37 \%$ yield).

Following the procedure (b) using 1 (218 mg, $2 \mathrm{mmol}), \mathbf{2 d}(88 \mathrm{mg}, 1 \mathrm{mmol})$, and $\mathrm{Et}_{3} \mathrm{~N}(101 \mathrm{mg}, 1 \mathrm{mmol}) \mathrm{a}$ mixture of products $\mathbf{3 d}$ and $\mathbf{4 d}$ was obtained. Subsequent purification gave $\mathbf{3 d}(\mathbf{7 9} \mathbf{m g}, 27 \%)$ and $\mathbf{4 d}(10 \mathrm{mg}$, $6 \%$ by ${ }^{1} \mathrm{H}$ NMR).

(Z)-2-[3-Cyano-4-(cyanomethylene)-5,5-dimethyl-4,5-dihydrofuran-2-yl]propan-2-yl isobutyrate (3d). Yellow oil; IR (film): 3094, 3066 (C=CH), 2994, 2976, 2937 (CH), 2223 and 2212 (C=N), 1713 (C=O), 1645, 1593, 1563 $(\mathrm{C}=\mathrm{C}) \mathrm{cm}^{-1} ;{ }^{1} \mathrm{H}$ NMR $\left(400.1 \mathrm{MHz}, \mathrm{CDCl}_{3}\right): \delta=5.09(\mathrm{~s}, 1 \mathrm{H},=\mathrm{CHCN}), 2.58(\mathrm{~m}, 1 \mathrm{H}, \mathrm{CH}), 1.71\left[\mathrm{~s}, 6 \mathrm{H},\left(\mathrm{CH}_{3}\right)_{2} \mathrm{C}-1\right]$, $\left.1.68\left[\mathrm{~s}, 6 \mathrm{H},\left(\mathrm{CH}_{3}\right)_{2} \mathrm{C}-5\right], 1.16\left[\mathrm{~d}, 6 \mathrm{H},\left(\mathrm{CH}_{3}\right)_{2} \mathrm{CH}\right], J 6.9 \mathrm{~Hz}\right] ;{ }^{13} \mathrm{C} \mathrm{NMR}\left(100.6 \mathrm{MHz}, \mathrm{CDCl}_{3}\right): \delta=182.4(\mathrm{C}-2), 175.7$

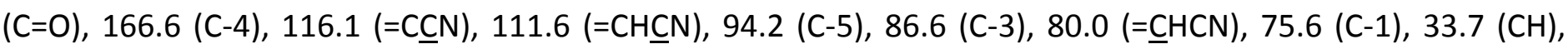
$25.0\left[\left(\underline{\mathrm{CH}}_{3}\right)_{2} \mathrm{C}-1\right], 24.4\left[\left(\mathrm{CH}_{3}\right)_{2} \mathrm{C}-5\right], 18.7\left[\left(\mathrm{CH}_{3}\right)_{2} \mathrm{CH}\right] ; \mathrm{MS}(\mathrm{EI}): \mathrm{m} / z$ (\%) = 288 (17) [M] ${ }^{+}, 201$ (12), 175 (19), 71 (68), 43 (100), 41 (18); Anal. Calcd for $\mathrm{C}_{16} \mathrm{H}_{20} \mathrm{~N}_{2} \mathrm{O}_{3}$ (288.34): C 66.65; H 6.99; N 9.72. Found: C 66.84; H 7.18; N 9.94.

5,5-Dimethyl-4-oxo-2-propyl-4,5-dihydro-3-furancarbonitrile (4d). White solid, mp 423-44 ${ }^{\circ} \mathrm{C}$; IR ( $\mathrm{KBr}$ ): 2986, 2940 (CH), 2229 (CN), 1723 (C=O), 1639, 1586, 1552 (C=C) cm ${ }^{-1} ;{ }^{1} \mathrm{H} \mathrm{NMR}\left(400.1 \mathrm{MHz} \mathrm{CDCl}_{3}\right): \delta=3.18(\mathrm{~m}, 1 \mathrm{H}$, $\mathrm{CH}), 1.44\left(\mathrm{~s}, 6 \mathrm{H}, 2 \mathrm{CH}_{3}\right), 1.34\left[\mathrm{~d}, 6 \mathrm{H},\left(\mathrm{CH}_{3}\right)_{2} \mathrm{CH}, J 6.9 \mathrm{~Hz}\right] ;{ }^{13} \mathrm{C} \mathrm{NMR}\left(100.6 \mathrm{MHz}, \mathrm{CDCl}_{3}\right): \delta=201.6(\mathrm{C}=0), 199.3$ (C-2), 111.1 (CN), 91.4 (C-5), 89.1 (C-3), $30.9(\mathrm{CH}), 22.4\left(2 \mathrm{CH}_{3}\right), 18.8\left[\left(\mathrm{CH}_{3}\right)_{2} \mathrm{CH}\right] ; \mathrm{MS}(\mathrm{EI}): m / z(\%)=180(13)$ $[\mathrm{M}+\mathrm{H}]^{+}, 179(82)[\mathrm{M}]^{+}, 178(19), 164$ (12), 136 (70), 136 (47), 108 (17), 94 (11), 93 (17), 92 (16), 71 (15), 69 (25), 67 (13), 66 (72), 59 (11), 58 (33), 43 (100), 42 (14), 41 (47), 39 (33); Anal. Calcd for $\mathrm{C}_{10} \mathrm{H}_{13} \mathrm{NO}_{2}$ (179.22): C, $67.02 ; H, 7.31 ; N, 7.82$. Found: $C, 67.23 ; H, 7.37 ; N, 8.20$.

3-Cyano-1,1-dimethyl-2-oxopropyl 2-methylpropanoate (5d). Light yellow oil; IR (film): 2979, 2935, 2882 (CH), $2261(\mathrm{CN}), 1730(\mathrm{C}=\mathrm{O}, \mathrm{COO}) \mathrm{cm}^{-1} ;{ }^{1} \mathrm{H}$ NMR (400.1 MHz, $\left.\mathrm{CDCl}_{3}\right): \delta=3.53\left(\mathrm{~s}, 2 \mathrm{H}, \mathrm{CH}_{2} \mathrm{CN}\right), 2.59(\mathrm{~m}, 1 \mathrm{H}, \mathrm{CH})$, $1.51\left(\mathrm{~s}, 6 \mathrm{H}, 2 \mathrm{CH}_{3}\right), 1.18\left[\mathrm{~d}, 6 \mathrm{H},\left(\mathrm{CH}_{3}\right)_{2} \mathrm{CH}, J 7.0 \mathrm{~Hz}\right] ;{ }^{13} \mathrm{C} \mathrm{NMR}\left(100.6 \mathrm{MHz}, \mathrm{CDCl}_{3}\right): \delta=196.4(\mathrm{C}=0), 176.8\left(\mathrm{CO}_{2}\right)$, $113.6(\mathrm{CN}), 82.7(\mathrm{C}-1), 33.7(\mathrm{CH}), 26.5\left(\underline{\mathrm{CH}}_{2} \mathrm{CN}\right), 23.0\left(2 \mathrm{CH}_{3}\right), 18.5\left[\left(\mathrm{CH}_{3}\right)_{2} \mathrm{CH}\right] ; \mathrm{MS}(\mathrm{EI}): \mathrm{m} / z(\%)=129(13)$ $\left[\mathrm{M}-\mathrm{C}(\mathrm{O}) \mathrm{CH}_{2} \mathrm{CN}\right]^{+}, 71(90), 59$ (34), 43 (100), 41 (36), 39 (14); Anal. Calcd for $\mathrm{C}_{10} \mathrm{H}_{15} \mathrm{NO}_{3}$ (197.23): C, 60.90; $\mathrm{H}$, 7.67; N, 7.10. Found: C 60.88; H 7.78; N 6.94.

\section{Reaction of 4-hydroxy-4-methyl-2-pentynenitrile (1) with 3-methylbutanoic acid (2e)}

Following the procedure (a) using 1 (131 mg, $1.2 \mathrm{mmol}), 2 \mathrm{e}(102 \mathrm{mg}, 1 \mathrm{mmol})$, and $\mathrm{Et}_{3} \mathrm{~N}(101 \mathrm{mg}, 1 \mathrm{mmol})$ in $\mathrm{MeCN}(5 \mathrm{~mL})$ a mixture of products $3 \mathbf{e}, \mathbf{4 e}$ and $\mathbf{5 e}$ was obtained. Purification: column chromatography $(1.0 \times$ $\left.40 \mathrm{~cm}, \mathrm{SiO}_{2}, \mathrm{Et}_{2} \mathrm{O}-\mathrm{C}_{6} \mathrm{H}_{14}, 1: 1\right)$; this gave 3 e $(15 \mathrm{mg}, 10 \%), 4 \mathrm{e}(40 \mathrm{mg}, 21 \%)$ and $\mathbf{5 e}(110 \mathrm{mg}, 90 \%$ pure as determined by ${ }^{1} \mathrm{H} N \mathrm{NMR}, 47 \%$ yield). 
Following the procedure (b) using $1(218 \mathrm{mg}, 2 \mathrm{mmol}), 2 \mathrm{e}(102 \mathrm{mg}, 1 \mathrm{mmol})$, and $\mathrm{Et}_{3} \mathrm{~N}(101 \mathrm{mg}, 1 \mathrm{mmol}) \mathrm{a}$ mixture of products $3 e, 4 e$ and $5 e$ was obtained. Subsequent purification gave $3 e(132 \mathrm{mg}, 44 \%), 4 e(27 \mathrm{mg}$, $14 \%$ ) and 5 e (45 mg, 90\% pure as determined by ${ }^{1} \mathrm{H}$ NMR, $19 \%$ yield).

(Z)-2-[3-Cyano-4-(cyanomethylene)-5,5-dimethyl-4,5-dihydrofuran-2-yl]propan-2-yl 3-methylbutanoate (3e). Light yellow oil; IR (film): 3047 (C=CH), 2984, 2964, 2935 (CH), 2876 (CH), 2217 (CN), 1740 (C=O), 1623, 1583 $(\mathrm{C}=\mathrm{C}) \mathrm{cm}^{-1} ;{ }^{1} \mathrm{H}$ NMR $\left(400.1 \mathrm{MHz}, \mathrm{CDCl}_{3}\right): \delta=5.10(\mathrm{~s}, 1 \mathrm{H},=\mathrm{CHCN}), 2.23\left(\mathrm{~m}, 2 \mathrm{H}, \mathrm{CH}_{2}\right), 2.07(\mathrm{~m}, 1 \mathrm{H}, \mathrm{CH}), 1.71[\mathrm{~s}$, $\left.6 \mathrm{H},\left(\mathrm{CH}_{3}\right)_{2} \mathrm{C}-1\right], 1.68\left[\mathrm{~s}, 6 \mathrm{H},\left(\mathrm{CH}_{3}\right)_{2} \mathrm{C}-5\right], 0.95\left[\mathrm{~d}, 6 \mathrm{H},\left(\mathrm{CH}_{3}\right)_{2} \mathrm{CH}, J 6.6 \mathrm{~Hz}\right] ;{ }^{13} \mathrm{C} \mathrm{NMR}(100.6 \mathrm{MHz}, \mathrm{CDCl} 3): \delta=182.5$ (C-2), 171.7 (C=O), 166.7 (C-4), 116.2 (=CN), 111.6 (=CHCN), 94.4 (C-5), 86.7 (C-3), 80.1 (=ㄷCN), 75.8 (C-1), $42.8\left(\mathrm{CH}_{2}\right), 25.5(\mathrm{CH}), 25.1\left[\left(\mathrm{CH}_{3}\right)_{2} \mathrm{C}-1\right], 24.5\left[\left(\mathrm{CH}_{3}\right)_{2} \mathrm{C}-5\right], 22.2\left[\left(\mathrm{CH}_{3}\right)_{2} \mathrm{CH}\right] ; \mathrm{MS}(\mathrm{El}): \mathrm{m} / \mathrm{z}(\%)=302(47)[\mathrm{M}]^{+}, 203$ (13), 202 (26), 201 (30), 200 (11), 175 (20), 85 (93), 69 (12), 57 (100), 43 (41), 41 (44), 39 (11); Anal. Calcd for $\mathrm{C}_{17} \mathrm{H}_{22} \mathrm{~N}_{2} \mathrm{O}_{3}$ (302.37): $\mathrm{C}, 67.53 ; \mathrm{H}, 7.33 ; \mathrm{N}, 9.26$. Found: $\mathrm{C}, 67.72 ; \mathrm{H}, 7.71 ; \mathrm{N}, 9.42$.

2-Isobutyl-5,5-dimethyl-4-oxo-4,5-dihydro-3-furancarbonitrile (4e). Light yellow oil; IR (film): 2967, 2937, 2877, 2229 (CN), 1724 (C=O), 1646, $1587(\mathrm{C}=\mathrm{C}) \mathrm{cm}^{-1} ;{ }^{1} \mathrm{H} \mathrm{NMR}\left(400.1 \mathrm{MHz}, \mathrm{CDCl}_{3}\right): \delta=2.64\left(\mathrm{~m}, 2 \mathrm{H}, \mathrm{CH}_{2}\right), 2.21$ $(\mathrm{m}, 1 \mathrm{H}, \mathrm{CH}), 1.45\left(\mathrm{~s}, 6 \mathrm{H}, 2 \mathrm{CH}_{3}\right), 1.04\left[\mathrm{~d}, 6 \mathrm{H},\left(\mathrm{C}_{3}\right)_{2} \mathrm{CH}, J 6.6 \mathrm{~Hz}\right] ;{ }^{13} \mathrm{C} \mathrm{NMR}\left(100.6 \mathrm{MHz}, \mathrm{CDCl}_{3}\right): \delta=199.0(\mathrm{C}=\mathrm{O})$, 197.1 (C-2), $111.3(\mathrm{CN}), 91.9(\mathrm{C}-3,5), 39.4\left(\mathrm{CH}_{2}\right), 22.2\left[\left(\mathrm{CH}_{3}\right)_{2} \mathrm{CH}\right], 27.2(\mathrm{CH}), 22.6\left(2 \mathrm{CH}_{3}\right) ; \mathrm{MS}(\mathrm{El}): \mathrm{m} / \mathrm{z}(\%)=193$ (53) [M] ${ }^{+}, 151$ (48),150 (14), 136 (70), 123 (17), 108 (11), 93 (12), 69 (12), 65 (15), 64 (12), 59 (19), 58 (11), 43 (100), 41 (45), 39 (25); Anal. Calcd for $\mathrm{C}_{11} \mathrm{H}_{15} \mathrm{NO}_{2}$ (193.24): C, 68.37; $\mathrm{H}, 7.82 ; \mathrm{N}, 7.25$. Found: C, 68.25; $\mathrm{H}, 7.47$; $\mathrm{N}, 7.50$.

3-Cyano-1,1-dimethyl-2-oxopropyl 3-methylbutanoate (5e). Light yellow oil; IR (film): 2963, 2933, 2879 (CH), $2261(\mathrm{CN}), 1732(\mathrm{C}=\mathrm{O}, \mathrm{COO}) \mathrm{cm}^{-1} ;{ }^{1} \mathrm{H} \mathrm{NMR}\left(400.1 \mathrm{MHz}, \mathrm{CDCl}_{3}\right): \delta=3.55\left(\mathrm{~s}, 2 \mathrm{H}, \mathrm{CH}_{2} \mathrm{CN}\right), 2.22\left(\mathrm{~m}, 2 \mathrm{H}, \mathrm{CH}_{2}\right), 2.21$ $(\mathrm{m}, 1 \mathrm{H}, \mathrm{CH}), 1.51\left(\mathrm{~s}, 6 \mathrm{H}, 2 \mathrm{CH}_{3}\right), 0.96\left[\mathrm{~d}, 6 \mathrm{H},\left(\mathrm{C}_{3}\right)_{2} \mathrm{CH}, J 6.6 \mathrm{~Hz}\right] ;{ }^{13} \mathrm{C} \mathrm{NMR}\left(100.6 \mathrm{MHz}, \mathrm{CDCl}_{3}\right): \delta=196.4(\mathrm{C}=\mathrm{O})$, $172.9\left(\mathrm{CO}_{2}\right), 113.6(\mathrm{CN}), 82.7(\mathrm{C}-1), 42.9\left(\mathrm{CH}_{2}\right), 26.6(\mathrm{CH}), 26.3\left(\underline{\mathrm{CH}}_{2} \mathrm{CN}\right), 23.1\left[\left(\mathrm{CH}_{3}\right)_{2} \mathrm{CH}\right], 22.2\left(2 \mathrm{CH}_{3}\right) ; \mathrm{MS}(\mathrm{EI})$ : $m / z(\%)=143(13)\left[\mathrm{M}-\mathrm{C}(\mathrm{O}) \mathrm{CH}_{2} \mathrm{CN}\right]^{+}, 85$ (100), 59 (17), 57 (86), 43 (13), 41 (34), 39 (12); Anal. Calcd for $\mathrm{C}_{11} \mathrm{H}_{17} \mathrm{NO}_{3}$ (211.26): C, 62.54; $\mathrm{H}, 8.11 ; \mathrm{N}, 6.63$. Found: $\mathrm{C} 62.84 ; \mathrm{H} 8.18 ; \mathrm{N} 6.63$.

\section{Acknowledgements}

The main results were obtained using the equipment of Baikal analytical center of collective using SB RAS.

\section{References}

1. Lee, Y.R.; Kang, K. Y.; Lee, G. J.; Lee, W. K. Synthesis 2003, 1977.

2. Garzino, F.; Méou, A.; Brun, P. Eur. J. Org. Chem. 2003, 8, 1410. https://doi.org/10.1002/ejoc.200390198

3. Kilroy, T. G.; O'Sullivan, T. P.; Guiry, P. J. Eur. J. Org. Chem. 2005, 4929.

https://doi.org/10.1002/ejoc.200500489

4. Bechthold, A. F.-W. In Bioorganic chemistry: deoxysugars, polyketides and related classes: synthesis, biosynthesis, enzymes. Part of the Topics in Current Chemistry, Vol. 188; Rohr, J.; Rohr, J.; Bechthold, A. F.W., Eds.; Springer: Berlin, Heidelberg, 1997, pp 205.

5. Bhat, S. V.; Nagasampagi, B. A.; Sivakumar, M. In Chemistry of Natural Products; Nagasampagi, B. A.; Sivakumar, M., Eds.; Springer Science \& Business Media: Berlin, Heidelberg, New York, 2005, pp 840.

6. Fraser-Reid, B. O.; Tatsuta, K.; Thiem, J., Eds. In Glycoscience: Chemistry and Chemical Biology I-III; Springer-Verlag: Berlin, Heidelberg, New York, 2012, pp 2872. 
7. Haung, T. T.; Kirsch, S. F. In Targets in Heterocyclic Systems: Chemistry and Properties, Vol. 13 ; Attanasi, O. A.; Spinelli, D., Eds.; Societa Chimica Italiana: Rome, 2009, p 57.

8. Egi, M.; Azechi, K.; Saneto, M.; Shimizu, K.; Akai, S. J. Org. Chem. 2010, 75, 2123. https://doi.org/10.1021/jo100048j

9. Poonoth, M.; Krause, N. J. Org. Chem. 2011, 76, 1934. https://doi.org/10.1021/jo102416e

10. Hou, X.-F.; Yao, S.; Mándi, A.; Kurtán, T.; Tang, C.-P.; Ke, C.-Q.; Li, X.-Q.; Ye, Y. Org. Lett. 2012, 14, 460. https://doi.org/10.1021/ol202915r

11. Cao, Z.; Zhang, R.; Meng, X.; Li, H.; Li, J.; Zhu, H.; Chen, G.; Suna, X.; You, J. RSC Adv. 2016, 6, 74582. https://doi.org/10.1039/C6RA14051C

12. Gottlieb, O. R. In New Natural Products and Plant Drugs with Pharmacological, Biological, or Therapeutical Activity; Wagner, H. K.; Wolff, P. M., Eds.; Springer-Verlag: Berlin, Heidelberg, Germany, 1987, pp 227.

13. Fraga, B. M. Nat. Prod. Rep. 1992, 9, 217. https://doi.org/10.1039/np9920900217

14. Merrit, A. T.; Ley, S. V. Nat. Prod. Rep. 1992, 9, 243. https://doi.org/10.1039/np9920900243

15. Benassi, R. In Comprehensive Heterocyclic Chemistry II, Vol. 2; Katritzky, A. R.; Rees, C. W.; Scriven, E. F. V.; Bird, C. W., Eds.; Elsevier: Oxford, U.K., 1996, 259.

16. Ward, R. S. Nat. Prod. Rep. 1999, 16, 75. https://doi.org/10.1039/a705992b

17. Jiang, Y.; Ma, D. Tetrahedron: Asymmetry 2002, 13, 1033. https://doi.org/10.1016/S0957-4166(02)00239-2

18. Son, S.; Fu, G. C. J. Am. Chem. Soc. 2007, 129, 1046. https://doi.org/10.1021/ja068344y

19. Bernard, A. M.; Frongia, A.; Piras, P. P.; Secci F.; Spiga, M. Org. Lett. 2005, 7, 4565. https://doi.org/10.1021/ol0514606

20. Chuang, C.-P.; Chen, K.-P.; Hsu, Y.-L.; Tsai, A.-I.; Liu, S.-T. Tetrahedron 2008, 64, 7511. https://doi.org/10.1016/i.tet.2008.05.122

21. Nakano, T.; Miyazaki, K.; Kamimura, A. J. Org. Chem. 2014, 79, 8103. https://doi.org/10.1021/jo5013042

22. Lee, Y. R.; Suk, J. Y. Tetrahedron 2002, 58, 2359. https://doi.org/10.1016/S0040-4020(02)00118-7

23. Wan, B.; Jiang, X.; Jia, G.; Ma, S. Eur. J. Org. Chem. 2012, 4373. https://doi.org/10.1002/ejoc.201200527

24. Naveen, T.; Kancherla, R.; Maiti, D. Org. Lett. 2014, 16, 5446. https://doi.org/10.1021/ol502688r

25. Tang, S.; Liu, K.; Long, Y.; Gao, X.; Gao, M.; Lei, A. Org. Lett. 2015, 17, 2404. https://doi.org/10.1021/acs.orglett.5b00912

26. McDonald, F. E.; Connolly, C. B.; Gleason, M. M.; Towne T. B.; Treiber, K. D. J. Org. Chem. 1993, 58, 6952. https://doi.org/10.1021/jo00077a006

27. Evans, D. A.; Sweeney, Z. K.; Rovis, T.; Tedrow, J. S. J. Am. Chem. Soc. 2001, 123, 12095. https://doi.org/10.1021/ja011983i

28. He, T.; Gao, P.; Qiu, Y.-F.; Yan, X.-B.; Liu, X.-Y.; Liang, Y.-M. RSC Adv. 2013, 3, 19913. https://doi.org/10.1039/c3ra44467h 
29. Chen, J.; Chen, C.; Chen, J.; Wang, G.; Qu, H. Chem. Commun. 2015, 51, 1356. https://doi.org/10.1039/C4CC08363F

30. Cacchi, S.; Fabrizi, G.; Goggiamani, A.; lazzetti, A.; Madec, D.; Polic, G.; Prestat. G. Org. Biomol. Chem. 2011, 9, 8233 .

https://doi.org/10.1039/c1ob06593a

31. Ishino, Y.; Nishiguchi, I.; Nakao, S.; Hirashima, T. Chem. Lett. 1981, 5, 641.

https://doi.org/10.1246/cl.1981.641

32. Lu, X.; Zhu, G.; Ma, S. Tetrahedron Lett. 1992, 33, 7205.

https://doi.org/10.1016/S0040-4039(00)60873-0

33. Philippot, K.; Devanne, D.; Dixneuf, P. H. J. Chem. Soc., Chem. Commun. 1990, 17, 1199. https://doi.org/10.1039/c39900001199

34. Le Paih, J.; De'rien, S.; Dixneuf, P. H. Chem. Commun. 1999, 15, 1437. https://doi.org/10.1039/a903805a

35. Wakabayashi, T.; Ishii, Y.; Murata, T.; Mizobe, Y.; Hidai, M. Tetrahedron Lett. 1995, 36, 5585. https://doi.org/10.1016/00404-0399(50)1067R-

36. Mal'kina, A. G.; Volostnykh, O. G.; Petrushenko, K. B.; Shemyakina, O. A.; Nosyreva, V. V.; Ushakov, I. A.; Trofimov, B. A. Tetrahedron 2013, 69, 3714. https://doi.org/10.1016/i.tet.2013.03.007

37. Mal'kina, A. G.; Volostnykh, O. G.; Stepanov, A. V.; Ushakov, I. A.; Petrushenko, K. B.; Trofimov, B. A. Synthesis 2013, 3435.

38. Trofimov, B. A.; Stepanov, A. V.; Mal'kina, A. G.; Volostnykh, O. G.; Shemyakina, O. A.; Ushakov, I. A. Synth. Commun. 2015, 45, 2718. https://doi.org/10.1080/00397911.2015.1103872

39. Mal'kina, A. G.; Shemyakina, O. A.; Stepanov, A. V.; Volostnykh, O. G.; Ushakov, I. A.; Sobenina, L. N.; Borodina, T. N.; Smirnov, V. I.; Trofimov, B. A. Synthesis 2016, 271.

40. Mal'kina, A. G.; Stepanov, A. V.; Sobenina, L. N.; Shemyakina, O. A.; Ushakov, I. A.; Smirnov, V. I.; Trofimov, B. A. Synthesis 2016, 1880.

41. Shemyakina, O. A.; Mal'kina, A. G.; Nosyreva, V. V.; Ushakov, I. A.; Trofimov, B. A. Arkivoc 2012,(viii), 319. http://dx.doi.org/10.3998/ark.5550190.0013.827

42. Shemyakina, O. A.; Stepanov, A. V.; Volostnykh, O. G.; Mal'kina, A. G.; Ushakov, I. A.; Trofimov, B. A. Russ. J. Org. Chem. 2014, 11, 1617. https://doi.org/10.1134/S107042801411013X

43. Landor, S. R.; Demetriou, B.; Grzeskowiak, R.; Pavey D. J. Organomet. Chem. 1975, 93, 129. https://doi.org/10.1016/S0022-328X(00)94152-4 